\title{
Optimisation des cycles combinés gaz/vapeur avec un ou deux niveaux de pression et chaudière à surface d'échange imposée
}

\author{
V.E. Cenusa' ${ }^{1}$ M. Feidt' ${ }^{2}$, R. Benelmir' ${ }^{2}$ et A. Badea' \\ 1 Faculté d'Énergétique, Université Polytechnique de Bucarest, 313, Splaiul Independentei, R 77206, secteur 6, Bucarest-Roumanie \\ 2 LEMTA, Université Henri Poincaré Nancy I, UMR 7563 (CNRS-INPL-UHP) 2, avenue de la Forêt de Haye, \\ BP 160, 54504 Vandoeuvre Cedex - France \\ e-mail : victor.cenusa@energ.pub.ro (cenusa_victor@yahoo.com); badea@study.energ.pub.ro \\ michel.feidt@ensem.inpl-nancy.fr - Riad.Benelmir@ensem.inpl-nancy.fr
}

\begin{abstract}
Résumé - Cet article présente l'optimisation des cycles combinés gaz/vapeur avec des chaudières récupératrices ayant un ou deux niveaux de pression pour la production de vapeur sans resurchauffe. L'objectif est de maximiser la puissance électrique produite, avec limitation de la surface totale de transfert de chaleur de la chaudière de récupération. La solution proposée est d'abord thermodynamique, mais elle tient aussi compte des aspects économiques par la diminution indirecte du coût. Les turbines à gaz étant choisies parmi les modèles extraits des catalogues industriels, l'objectif devient celui de maximiser la puissance électrique générée par la turbine à vapeur. Le modèle mathématique présenté couvre l'ensemble des installations du cycle combiné dans leurs couplages et met en évidence la présence d'une pression ou d'une paire de pressions pour lesquelles on obtient l'optimum thermoéconomique. Le travail contient, aussi, une analyse et une optimisation économique de la chaudière de récupération à un niveau de pression, dans les mêmes hypothèses.
\end{abstract}

\begin{abstract}
Optimising Combined Cycle with One or Two Steam Pressure Levels and Heat Recovery Generator with Imposed Transfer Area - The paper treats about optimising the combined cycles with heat recovery systems having one or two steam pressure levels, without reheat. The thermodynamic target is maximising the electrical output without growing the steam generators heat transfer area. This thermodynamical approach takes into consideration the economical aspect by this kind of indirect investment restriction. The gas turbines being choosed from the producer's directories, the target became maximising the electrical output generated by the steam turbine. The mathematical model covers the whole combined cycle installation's in their coupling. It put into evidence the presence of one optimal pressure or a couple of two optimal pressures which offer the thermoeconomical optimum. In the paper the authors make an economical optimisation for the heat recovery steam generator with one pressure level.
\end{abstract}




\section{NOMENCLATURE}

BP basse pression

C coût spécifique de l'échangeur de chaleur $\left(u m \cdot \mathrm{m}^{-2}\right)$

CC cycle combiné

$\mathrm{Cd}$ condenseur

$\mathrm{CR}$ chaudière de récupération

ECO économiseur

$\mathrm{f}_{\mathrm{SC}} \quad$ coefficient $\left(=\mathrm{k}_{\mathrm{ECO}} / \mathrm{k}_{\mathrm{SC}}\right)(-)$

GE générateur électrique (alternateur)

$\mathrm{h}$ enthalpie massique $(\mathrm{kJ} / \mathrm{kg})$

HP haute pression

I investissement (um)

$\mathrm{k} \quad$ coefficient global d'échange de chaleur $\left(\mathrm{kW} \cdot \mathrm{m}^{-2} \cdot{ }^{\circ} \mathrm{C}^{-1}\right)$

$\mathrm{p}$ pression (bar)

$\mathrm{P} \quad$ puissance $(\mathrm{kW})$

PA pompe d'alimentation

$\mathrm{P}_{\text {int }} \quad$ puissance interne de la TV $(\mathrm{kW})$

PI le quota de l'investissement d'échangeur par rapport à l'investissement total de CR

PS le quota de surface d'échange par rapport à la surface totale d'échange de la CR

$\dot{\mathrm{Q}} \quad$ puissance thermique $(\mathrm{kW})$

RC le rapport entre le coût spécifique de surchauffeur et de l'économiseur

S la surface d'échange de chaleur $\left(\mathrm{m}^{2}\right)$

SC surchauffeur

$\mathrm{t}$ température $\left({ }^{\circ} \mathrm{C}\right)$

TG installation de turbine à gaz

TV turbine à vapeur

VAP système vaporisateur (bouilleur)

1 .. 17, 1B .. 9B, 1H .. 9H, 1Hi points caractéristiques

$1 \mathrm{p}$ un niveau de pression pour la production des vapeurs

$2 \mathrm{p}$ deux niveaux de pression pour la production des vapeurs.

\section{Grecques}

$\Delta \mathrm{P}_{\text {alt }} \quad$ pertes en alternateur $(\mathrm{kW})$

$\Delta \mathrm{P}_{\text {mec }}$ pertes mécaniques $(\mathrm{kW})$

$\Delta \mathrm{t}_{\mathrm{mln}} \quad$ la différence moyenne logarithmique de température $\left({ }^{\circ} \mathrm{C}\right)$.

\section{Indices}

b bornes d'alternateur

el électrique

j surface d'échange de chaleur de la CR

j1 les surfaces d'échange de chaleur des ECO et des VAP

j2 les surfaces d'échange de chaleur des surchauffeurs.

\section{INTRODUCTION}

Une série des travaux de spécialité présente le calcul et l'optimisation du cycle combiné avec les suivantes hypothèses: la différence minimale de température (le pincement) («pinch point ») [1] entre les deux fluides, dans la chaudière de récupération, constante [2-9] et la différence entre la température de saturation à la pression du ballon et la température de l'eau à la sortie d'économiseur (point d'approche) («approach point») [1] nulle. Les chaudières ainsi définies sont différentes du point de vue volume de métal utilisé et des surfaces d'échange de chaleur, ce qui fait que l'optimum thermodynamique obtenu est loin de l'optimum économique. D'autre part, une analyse économique exacte ne peut pas se faire que si on connaît une série de données concrètes en ce qui concerne les solutions techniques et les valeurs utilisées pour le dimensionnement de la chaudière. Ceci n'est pas possible que dans une analyse de cas.

Tenant compte de ce désavantage de la méthode classique du pincement, ont été développés des méthodes alternatives pour l'optimisation thermoéconomique des paramètres de fonctionnement de la chaudière de récupération $[10,11]$ ou une optimisation thermoéconomique des cycles combinés en utilisant un algorithme génétique [12].

Aussi, [13] a proposé l'utilisation d'une «différence de température moyenne logarithmique équivalente » pour atténuer les inconvénients de la méthode «pinch-point» dans les cas de CR avec un seul niveau de pression de production de vapeur et sans surchauffeurs (générateur de vapeur de centrale nucléaire).

La solution proposée dans ce travail est d'abord thermodynamique, mais elle tient compte des aspects économiques par la considération d'une surface totale de transfert de chaleur constante pour la chaudière de récupération. Une telle supposition permet la détermination d'une valeur technique optimisée beaucoup plus proche de l'optimum économique, sans entrer dans des grands détails et tient compte de la majorité des tendances contraires, qui déterminent l'existence d'un optimum technique avec contraintes. Les diverses chaudières équivalentes du point de vue de la surface de transfert de chaleur n'auront pas la même différence minimale température, celle-ci résultant du programme de calcul itératif qui respecte les contraintes et maximise les fonctions objectifs.

\section{SCHÉMAS D'INSTALLATION}

Le schéma d'installation pour le CC $1 \mathrm{p}$ est présenté sur la figure 1 et le schéma d'installation pour le CC $2 p$ sur la figure 2.

Du point de vue constructif, on a choisi, pour CR $2 p$, l'emplacement en parallèle de l'économiseur basse pression $\left(\mathrm{ECO}_{\mathrm{BP}}\right)$ avec une partie de l'économiseur haute pression $\left(\mathrm{ECO}_{\mathrm{HP}_{-}}\right)$. Ce schéma est plus utilisé que le schéma avec 


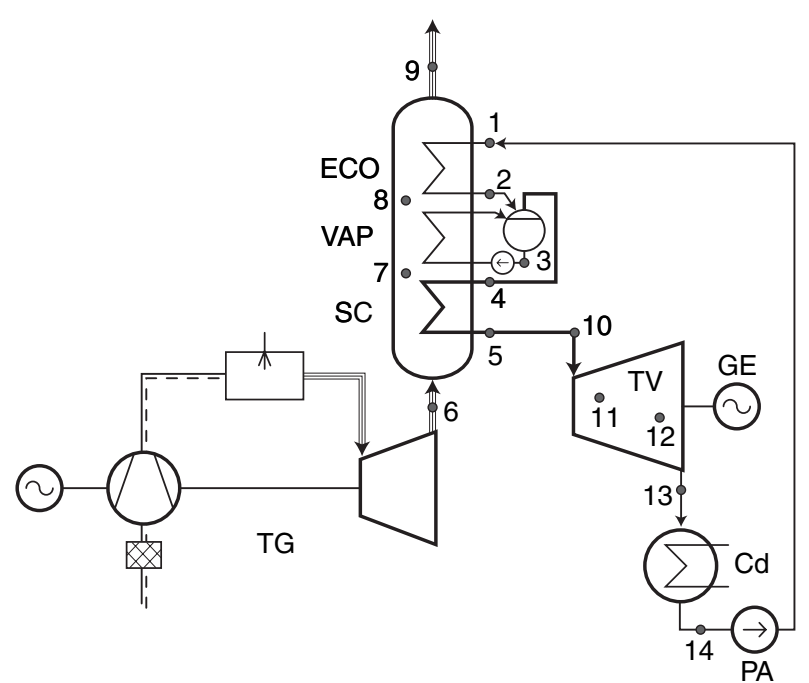

Figure 1

CC 1p: le schéma d'installation.

CC 1p: the system layout.

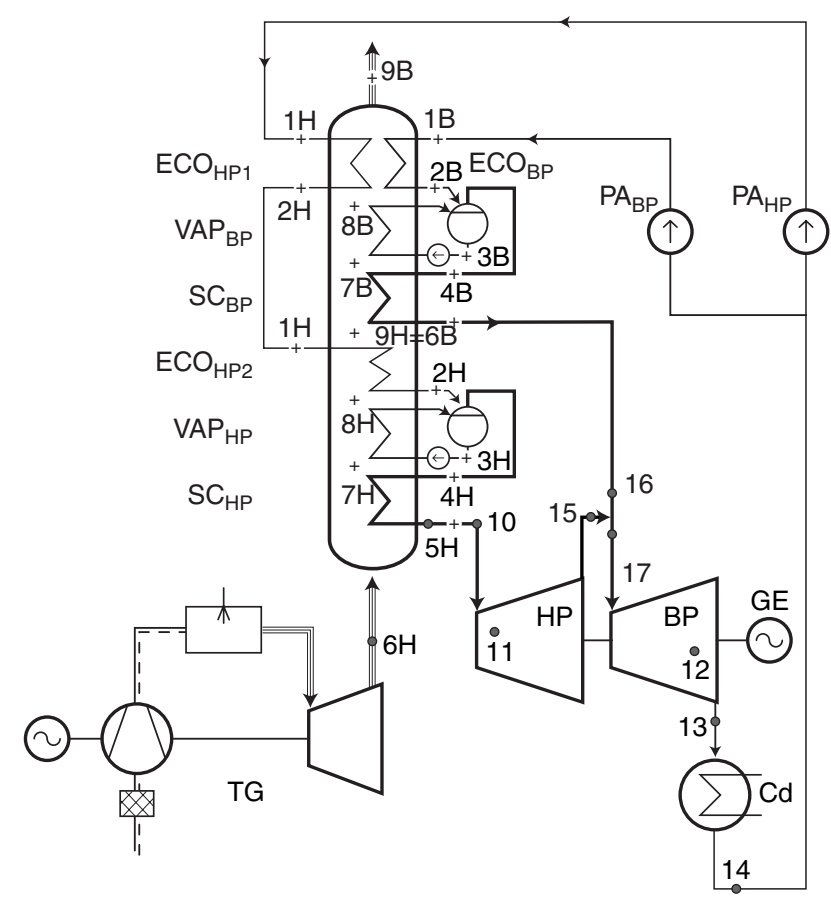

Figure 2

CC 2p: le schéma d'installation.

$C C 2 p$ : the system layout.

toutes les surfaces d'échange de chaleur en cascade ou série $[14,15]$ du fait de pertes exergétiques plus petites. Pour avoir une température d'eau très petite à l'entrée de la CR, on propose les modalités suivantes pour le dégazage : au condenseur, dégazage chimique, au ballon basse pression pour CR 2p $[15,16]$. Dans le CC 2p (fig. 2), la vapeur de basse pression produite par la $\mathrm{CR}$ rencontre la vapeur sortie de la section HP de la TV et le débit de vapeur résultant entre dans la section BP de la TV.

Les diagrammes $\mathrm{t}\left({ }^{\circ} \mathrm{C}\right)-\mathrm{h}(\mathrm{kJ} / \mathrm{kg})$ (ou h représente l'enthalpie des fumées) correspondant pour le CR 1p, respectivement pour le CR $2 p$ et les points caractéristiques des installations sont présentés sur les figures 3 et 4 .

\section{MODÈLE MATHÉMATIQUE DU CC}

\subsection{Chaudière de récupération}

\subsubsection{Hypothèses}

$[16,17]$ énoncent et expliquent les hypothèses de calcul utilisées pour le modèle mathématique de la CR. On rappelle, succinctement, les hypothèses principales :

- CR sans postcombustion;

- Surface totale d'échange de chaleur (SCR $\left(\mathrm{m}^{2}\right)$ ) constante ;

- Coefficient global d'échange de chaleur de l'économiseur $\left(\mathrm{k}_{\mathrm{ECO}}\left(\mathrm{kW} / \mathrm{m}^{2} /{ }^{\circ} \mathrm{C}\right)\right)$ constant; il en résulte que le produit $\mathrm{S}_{\mathrm{CR}} \cdot \mathrm{k}_{\mathrm{ECO}}=$ constant (paramètre);

- Coefficients globaux d'échange de chaleur des économiseur (des économiseurs) et du bouilleur (des bouilleurs) sont pratiquement égaux $\left(=\mathrm{k}_{\mathrm{ECO}}\right)[13,18-20]$;

- Coefficients globaux d'échange de chaleur des deux surchauffeurs, dans le cas CR $2 \mathrm{p}$, sont égaux $\left(=\mathrm{k}_{\mathrm{SC}}\right)[18-$ 20];

- $\mathrm{k}_{\mathrm{ECO}}>\mathrm{k}_{\mathrm{SC}}$ (coefficient global d'échange de chaleur réduit sur la partie vapeur surchauffée [19]; on pose $\mathrm{f}_{\mathrm{SC}}=\mathrm{k}_{\mathrm{ECO}}$ / $\mathrm{k}_{\mathrm{SC}}>1$ (paramètre);

- L'enthalpie et l'entropie des fumées sont fonction des température, pression et composition molaire des fumées $\left(\mathrm{N}_{2}, \mathrm{O}_{2}, \mathrm{H}_{2} \mathrm{O}, \mathrm{CO}_{2}\right)[21]$;

- $\mathrm{t}_{6}-\mathrm{t}_{5}=$ imposé (figs 1 et 3 ) ; $\mathrm{t}_{6 \mathrm{~B}}-\mathrm{t}_{5 \mathrm{~B}}=\mathrm{t}_{6 \mathrm{H}}-\mathrm{t}_{5 \mathrm{H}}=$ imposés (figs 2 et 4 ) ;

- Pincement («pinch-point $)=\mathrm{t}_{8 \mathrm{~B}}-\mathrm{t}_{3 \mathrm{~B}}=\mathrm{t}_{8 \mathrm{H}}-\mathrm{t}_{3 \mathrm{H}}$ (fig. 4) [22];

- Point d'approche («approach-point $»)=t_{3 B}-t_{2 B}=t_{3 H}-t_{2 H}$ (fig. 4).

\subsubsection{Modèle}

$[16,17,23]$ décrivent le modèle utilisé et la validation de celui-ci pour la $\mathrm{CR}$. On fait une synthèse sur le modèle mathématique, en spécifiant que cette fois-ci on a intégré le calcul des PA pour déterminer les enthalpies d'eau à l'entrée dans la CR.

Pour déterminer la distribution des températures dans la $\mathrm{CR}$ on a besoin du pincement. Celui-ci étant une inconnue pour le modèle, la façon de le calculer est itérative. Le pincement choisi est celui pour lequel la différence entre $\mathrm{S}_{\mathrm{CR}} \cdot \mathrm{k}_{\mathrm{ECO}}$ imposé par l'utilisateur et le produit calculé par le programme (3) tend vers zéro. 


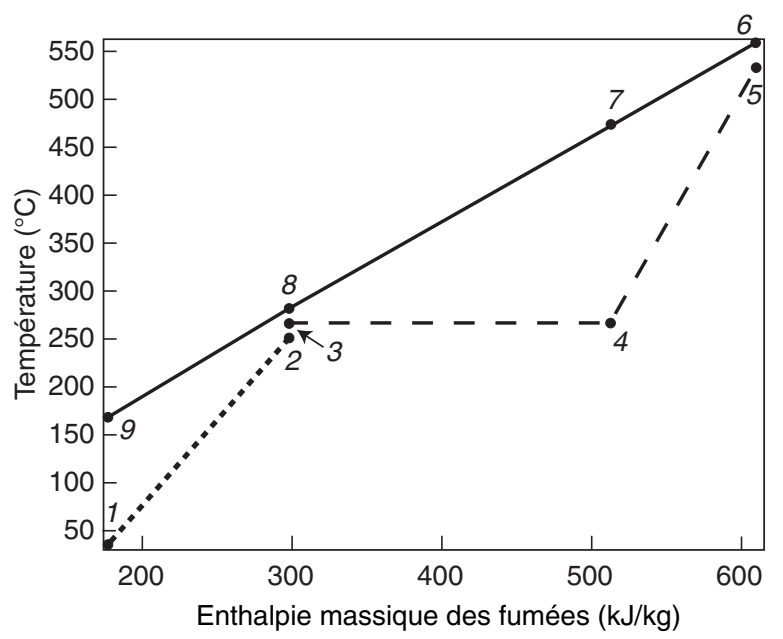

Figure 3

Diagramme $\mathrm{t}\left({ }^{\circ} \mathrm{C}\right)-\mathrm{h}(\mathrm{kJ} / \mathrm{kg})$, pour $\mathrm{CR} 1 \mathrm{p}$; points caractéristiques.

The $t\left({ }^{\circ} \mathrm{C}\right)-h(\mathrm{~kJ} / \mathrm{kg})$ diagram for the HRSG 1p: the characteristic points.

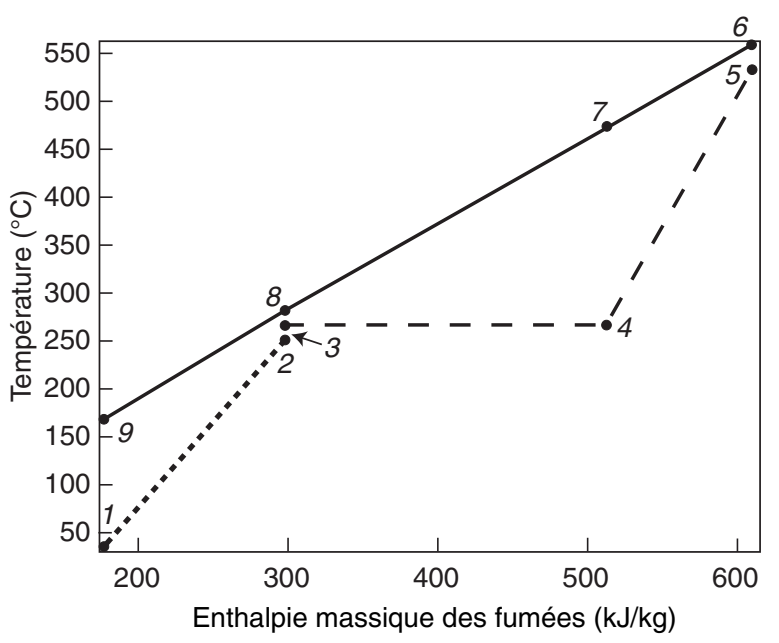

Figure 4

Diagramme $\mathrm{t}\left({ }^{\circ} \mathrm{C}\right)-\mathrm{h}(\mathrm{kJ} / \mathrm{kg})$, pour $\mathrm{CR} 2 \mathrm{p}$; points caractéristiques.

The $t\left({ }^{\circ} \mathrm{C}\right)-h(\mathrm{~kJ} / \mathrm{kg})$ diagram for the HRSG $2 \mathrm{p}$ : the characteristic points.
On calcule les différences de températures moyennes logarithmiques $\left(\Delta t_{m \ln , j}\left({ }^{\circ} \mathrm{C}\right)\right)$ [18] pour chacune des surfaces d'échange de chaleur (,j’). Les puissances thermiques $\left(\mathrm{Pt}_{\mathrm{j}}\right.$ $(\mathrm{kW}))$ transférées par ces surfaces peuvent être écrites en utilisant la relation générique suivante:

$$
\dot{Q}_{j}=k_{j} S_{j} \Delta t_{m \ln , j}
$$

où :

,j " fait référence à chaque surface d'échange de chaleur

$k$ coefficient global d'échange de chaleur $\left(\mathrm{kW} \cdot \mathrm{m}^{-2} \cdot{ }^{\circ} \mathrm{C}^{-1}\right)$

$S$ la surface d'échange de chaleur $\left(\mathrm{m}^{2}\right)$.

De (1) on obtient la relation générique de calcul pour chaque surface d'échange de chaleur $\left(S_{j}\right)$, ainsi que la relation de calcul de la surface totale d'échange de chaleur de la CR $\left(S_{C R}\right)$ sous la forme :

$$
S_{C R}=\sum_{j} S_{j}=\sum_{j} \frac{\dot{Q}_{j}}{k_{j} \Delta t_{m \ln , j}}
$$

Tenant compte des hypothèses de calcul considérées, la relation (2), par multiplication avec $\mathrm{k}_{\mathrm{ECO}}$ devient :

$$
\begin{aligned}
& S_{C R} k_{E C O}=k_{E C O} \sum_{j} \frac{\dot{Q}_{j}}{k_{j} \Delta t_{m \ln , j}} \\
& =\sum_{j 1} \frac{\dot{Q}_{j 1}}{\Delta t_{m \ln , j 1}}+f_{S C} \sum_{j 2} \frac{\dot{Q}_{j 2}}{\Delta t_{m \ln , j 2}}
\end{aligned}
$$

où avec,$j 1$ " on note les surfaces d'échange de chaleur des économiseurs et des systèmes de vaporisation et avec,$j 2$ " les surfaces d'échange de chaleur des surchauffeurs.
Les variables résultant du calcul itératif fait au niveau de la CR permettent d'établir les paramètres d'entrée dans la TV.

\subsection{Cycle à vapeur}

\subsubsection{Hypothèses}

- Les pertes de pression et d'enthalpie sont imposées pour les tuyaux de liaison entre la CR et la TV :

. $\mathrm{p}_{10}=0,9 \mathrm{p}_{5} ; \mathrm{p}_{10}=0,9 \mathrm{p}_{5 \mathrm{H}} ; \mathrm{p}_{16}=0,9 \mathrm{p}_{5 \mathrm{~B}}$;

. $\mathrm{h}_{10}=\mathrm{h}_{5}-4 ; \mathrm{h}_{10}=\mathrm{h}_{5 \mathrm{H}}-4 ; \mathrm{h}_{16}=\mathrm{h}_{5 \mathrm{~B}}-4$.

- L'étage de réglage (du débit de vapeur) pour la TV (entre les points 10 et 11 , voir figs 1 et 2 ) peut être de type «Rateau» ou «Curtis». L'étage «Curtis» a deux séries d'ailettes mobiles pour avoir une grande détente. Par comparaison, l'étage de réglage de type «Rateau » à une seule série d'ailettes mobiles, mais il a un meilleur rendement isentropique que l'étage «Curtis». Néanmoins l'étage «Curtis » rend la TV plus compacte.

- La pression dans l'espace entre l'étage de réglage et les étages de pression (point 11, voir figs 1 et 2) est considérée: $\mathrm{p}_{11}=0,85 \mathrm{p}_{10}$. Cette hypothèse est réaliste pour l'étage « Rateau ».

- Dans le CC 2p (fig. 2), la pression de la vapeur à la sortie de la section HP de la TV $\left(\mathrm{p}_{15}\right)$ est la même que la pression de la vapeur de base produite par la CR à l'entrée dans la section BP de la TV $\left(\mathrm{p}_{16}\right): \mathrm{p}_{15}=\mathrm{p}_{16}=\mathrm{p}_{17}$.

- Les rendements isentropiques pour l'étage de réglage et les étages de pression de chaque section de la TV (HP et $\mathrm{BP})$ ont été calculés en utilisant des formules semiempiriques conformément à [24]. 
- La vitesse axiale de la vapeur à la sortie de chaque section de la TV a été fixée (100 m/s pour HP et $200 \mathrm{~m} / \mathrm{s}$ pour BP).

- Les propriétés thermodynamiques du fluide sont déterminées conformément à [25].

- Le rendement isentropique des PA est imposé : $75 \%$.

- Les produits des rendements de transmission de la puissance aux PA sont imposés (le rendement du moteur électrique de la PA, le rendement mécanique) : $97 \%$.

- Au condenseur on connaît la température de condensation de la vapeur (ou la pression de condensation).

\subsubsection{Modèle}

Les pressions pour le circuit eau/vapeur sont connues. Elles sont dues aux hypothèses et aux schémas considérés.

Pour la TV on a les dépendances suivantes pour les rendements isentropiques [24]:

- Le rendement isentropique pour l'étage de réglage est fonction de la pression, du volume massique à l'entrée dans l'étage et du débit massique de la vapeur.

- Le rendement isentropique pour les étages de pression de la section HP de la TV dépend du débit volumique moyen de la vapeur et de la détente isentropique dans la section de la turbine ; le calcul de ce rendement est itératif.

- Le rendement isentropique pour les étages de pression de la section BP de la TV dépend de la détente isentropique dans la section BP. Le rendement isentropique est corrigé (par multiplication), pour la zone de détente située sur la courbe de vapeurs saturées (titre $=1$ ), avec un facteur qui tient compte de l'effet négatif de l'humidité. Pour la zone de détente située sous la courbe de vapeur saturée on utilise un calcul itératif pour déterminer l'intersection entre la détente et cette courbe.

Les enthalpies réelles tiennent compte aussi des pertes par énergie cinétique résiduelle à la sortie de chaque section de la TV.

Les rendements mécanique et d'alternateur sont calculés en utilisant des fonctions d'interpolation pour les pertes dans l'alternateur $\left(\Delta \mathrm{P}_{\text {alt }}(\mathrm{kW})\right)$ et mécaniques $\left(\Delta \mathrm{P}_{\text {mec }}(\mathrm{kW})\right)$. Les graphiques de [24], à l'origine des interpolations, sont donnés pour les turbines à vapeur, dans les modes suivants :

$$
\begin{gathered}
\ln \left(\Delta \mathrm{P}_{\text {mec }}\right)=\text { fonction }\left(\ln \left(\mathrm{P}_{\text {int }}\right)\right) \\
\ln \left(\Delta \mathrm{P}_{\text {alt }}\right)=\text { fonction }\left(\ln \left(\mathrm{P}_{\mathrm{b}}\right)\right)
\end{gathered}
$$

Après l'interpolation on obtient les pertes estimées (6) et (7) $(\mathrm{kW})$, qu'on utilise pour l'estimation des rendements; le calcul pour les pertes en alternateur étant itératif :

$$
\begin{aligned}
& \Delta P_{\text {mec }}=e^{\left(-0,0111 \ln ^{2}\left(P_{\text {int }}\right)+0,83 \ln \left(P_{\text {int }}\right)-1,7318\right)} \\
& \Delta P_{\text {alt }}=e^{\left(0,0365 \ln ^{2}\left(P_{b}\right)-0,0253 \ln \left(P_{b}\right)+2,7366\right)}
\end{aligned}
$$

On calcule la puissance aux bornes de l'alternateur $\mathrm{P}_{\mathrm{b}}$ $(\mathrm{kW})$ et la puissance électrique semi-nette pour le cycle à vapeur $\left(\mathrm{P}_{\mathrm{el}}\right.$ semi-nette $)(\mathrm{kW})$ par la différence entre $\mathrm{P}_{\mathrm{b}}$ et la puissance reçue par les PA $(\mathrm{kW})$.

\section{MODÉLISATION ET OPTIMISATION DU CC}

La fonction objectif est la puissance électrique produite par le cycle combiné. Lorsque les turbines à gaz sont choisies parmi les types existant dans les catalogues des manufacturiers ; la fonction objectif est la puissance électrique produite par la turbine à vapeur.

Dans le cas du cycle combiné avec chaudière de récupération à un niveau de pression, pour la production de vapeur, le paramètre optimisé est la pression de production de vapeur dans la chaudière, alors que pour le cycle combiné avec chaudière de récupération à deux niveaux de pression, l'optimisation se fait sur deux paramètres simultanément : la basse pression et la haute pression.

Pour l'optimisation on a deux options en ce qui concerne la puissance électrique, qui peut être :

- aux bornes d'alternateur ou

- «semi-nette», en considérant que le principal consommateur de service interne de la centrale est la PA.

On a analysé l'influence de deux types de turbine à gaz [26], de fabrication General Electric, sur les valeurs optimisées :

- PG9231(EC) de type «heavy duty » (le débit des fumées de $508 \mathrm{~kg} . \mathrm{s}^{-1}$, la température des fumées à la sortie de la turbine de $558^{\circ} \mathrm{C}$ );

- LM2500+ de type «aerodérivative » (le débit des fumées de $83 \mathrm{~kg} . \mathrm{s}^{-1}$, la température des fumées à la sortie de la turbine de $510^{\circ} \mathrm{C}$ ).

La composition molaire des fumées pour ces turbines à été calculée a l'aide du programme de calcul qui réalise la simulation du fonctionnement des TG en partant des données du catalogue [27].

En pratique, on utilise, plus spécialement pour des turbines à gaz de moyenne puissance, deux ou trois TG. Chaque TG a une CR correspondante et le débit de vapeur sortant des CR est collecté dans une seule TV, augmentant ainsi le rendement isentropique de la TV et aussi les performances du cycle à vapeur.

Dans la figure 5 on observe l'influence du nombre de TG sur le rendement isentropique pour les étages de pression de la section HP de la TV pour les TG considérées, dans le cas d'optimisation du CC $2 \mathrm{p}$, en maintenant $\mathrm{p}_{5 \mathrm{~B}}$ constante (5 bar).

Le programme de calcul pour le CC 1p est divisé en deux programmes indépendants.

Ceux-ci :

- Modélisent le fonctionnement du CC. Dans ce programme l'utilisateur introduit la valeur de la pression de production de la vapeur $\left(\mathrm{p}_{5}\right)$. 


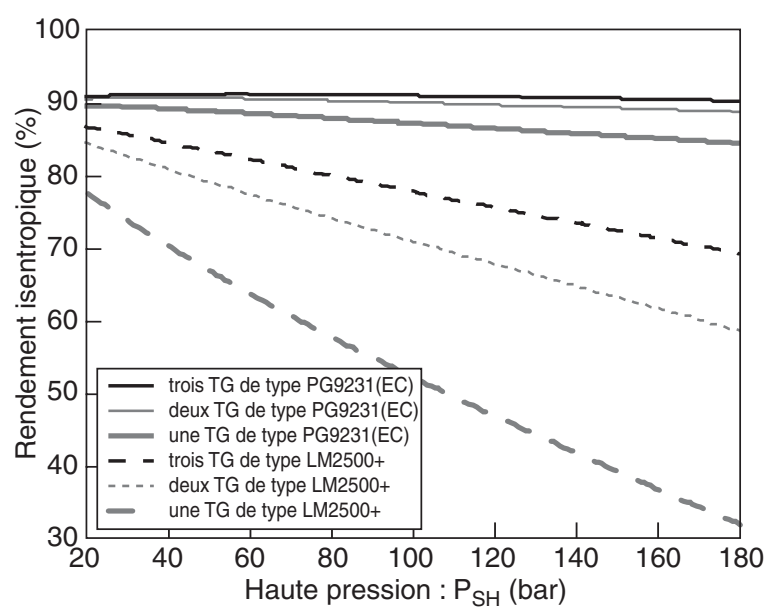

Figure 5

$\mathrm{CC} 2 \mathrm{p}$ : Variation du rendement isentropique pour la section HP de la TV fonction de $\mathrm{p}_{5 \mathrm{H}}$ et du nombre de TG.

CC 2p: The isentropic efficiency for the HP part of TV depending on $p_{5 H}$ and the gas turbines number.

- Optimisent le CC en fonction de la pression de la vapeur à la sortie du $\mathbf{C R}\left(\mathbf{p}_{5}\right)$. Le programme met en évidence la pression optimale ( $\mathbf{p}_{5}$ optimum $)$ pour laquelle se maximise la puissance électrique produite par la turbine à vapeur (fig. 6).

Le programme pour le CC $2 p$ est structuré en trois parties principales. Pratiquement il contient trois programmes indépendants. Ceux-ci:

- Modélisent le fonctionnement du CC. Dans ce programme l'utilisateur introduit les valeurs pour les deux pressions de production des vapeurs $\left(\mathrm{p}_{5 \mathrm{H}}\right.$ et $\left.\mathrm{p}_{5 \mathrm{~B}}\right)$.

- Optimisent le CC en fonction de la haute pression de la vapeur à la sortie du $\mathbf{C R}\left(\mathbf{p}_{5 \mathbf{H}}\right)$. La pression $\mathrm{p}_{5 \mathrm{~B}}$ est introduite par l'utilisateur. Le programme met en évidence la pression optimale $\left(\mathbf{p}_{5 \mathbf{5} \text { optimum }}\right)$ pour laquelle la puissance électrique produite par la turbine à vapeur est maximum (fig.7). La valeur de $\mathrm{p}_{5 \mathrm{~B}}$ utilisée pour déterminer la figure 7 est 5 bar.

- Optimisent le $\mathrm{CC}$ en fonction des deux paramètres simultanés $\left(\mathbf{p}_{\mathbf{5 H} \text { _optimum }}\right.$ et $\left.\mathbf{p}_{\mathbf{5 B}}\right)$ mettant en évidence la paire des pressions optimales $\left(\mathbf{p}_{5 \mathbf{5} \_ \text {optimum }}, \mathbf{p}_{\mathbf{5 B} \_ \text {optimum }}\right.$ ) pour laquelle on obtient un maximum maximorum de la puissance électrique produite par la turbine à vapeur (fig. 8).

\section{ANALYSES DES RÉSULTATS D'OPTIMISATION DU CC}

L'introduction de plusieurs niveaux de pression de production de vapeur réduit les dégradations exergétiques dans la CR (figs 3 et 4) en augmentant l'exergie transférée sur le cycle à vapeur [17] et accroissant ainsi la puissance électrique (brute ou nette) produite par la TV (figs 6 à 8). Le maximum maximorum des puissances électriques nettes s'obtient pour chacun des deux types de TG (figure 8a respectivement $8 b$ ). La courbe continue croissante qui apparaît sur les figures $8 \mathrm{a}$ et $8 \mathrm{~b}$ représente la variation de la haute pression optimale $\left(\mathrm{p}_{5 \mathrm{H} \_ \text {optimum }}\right)$ (l'ordonnée de droite) en fonction de la basse pression $\left(\mathrm{p}_{5 \mathrm{~B}}\right)$. Les valeurs des pressions $\mathrm{p}_{5 \mathrm{H} \_o p t i m u m}$ sont celles auxquelles on a maximisé la $\mathrm{P}_{\mathrm{el}}$ seminette à la pression $\mathrm{p}_{5 \mathrm{~B}}$ de l'abscisse (voir fig. 7). Ainsi, la courbe de variation de puissance électrique semi-nette maximale de la figure 8 est fonction des deux variables : $\mathrm{p}_{5 \mathrm{H} \_ \text {optimum }}\left(\mathrm{p}_{5 \mathrm{H}}\right.$ étant déjà une pression optimisée $)$ et $\mathrm{p}_{5 \mathrm{~B}}$. La pression $\mathrm{p}_{5 \mathrm{~B}}$ à laquelle se maximise $\mathrm{P}_{\mathrm{el}}$ semi-nette maximale (fig.8) (fonction de $\mathrm{p}_{5 \mathrm{H} \_ \text {optimum }}$ et $\mathrm{p}_{5 \mathrm{~B}}$ ) devient $\mathrm{p}_{5 \mathrm{~B} \_ \text {optimum }}$. La
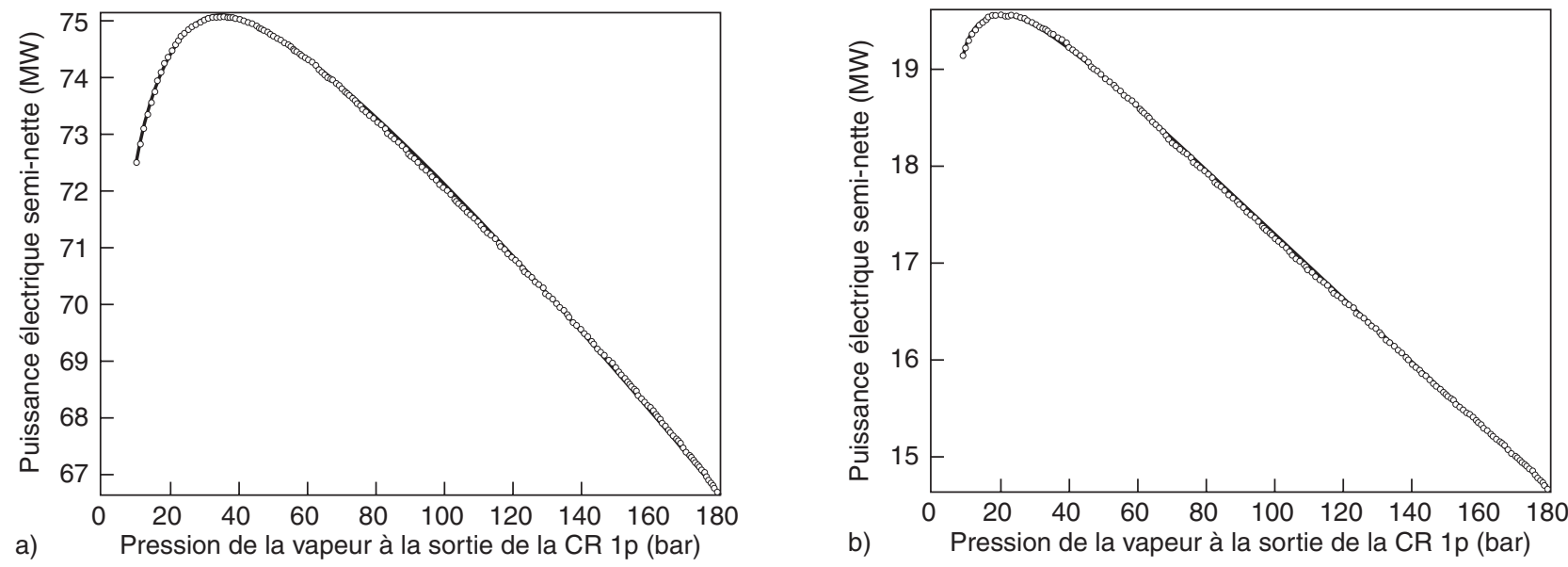

Figure 6

Optimisation CC 1p : $\mathrm{P}_{\mathrm{el}}$ semi-nette = fonction $\left(\mathrm{p}_{5 \mathrm{H}}\right)$. a) TG : une TG de type PG9231(EC); b) TG : deux TG de type LM2500+.

The CC 1p optimising: $P_{e l}$ output depending on $p_{5 H}$. 

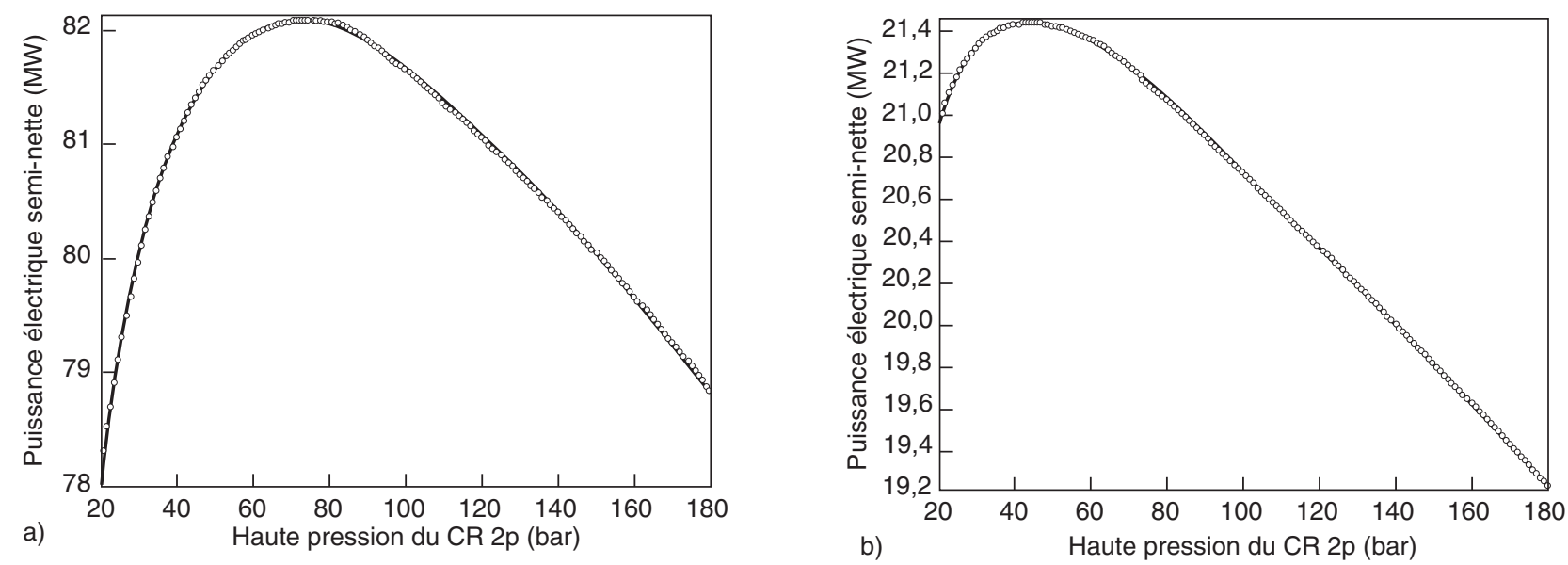

Figure 7

Optimisation CC $2 p: \mathrm{P}_{\mathrm{el}}$ semi-nette $=$ fonction $\left(\mathrm{p}_{5 \mathrm{H}}\right)$ pour $\mathrm{p}_{5 \mathrm{~B}}=5$ bar. a) TG : une TG de type PG9231(EC); b) TG : deux TG de type LM2500+.

The CC $2 p$ optimising: $P_{e l}$ output depending on $p_{5 H}$ for $p_{5 L}=5$ bar.
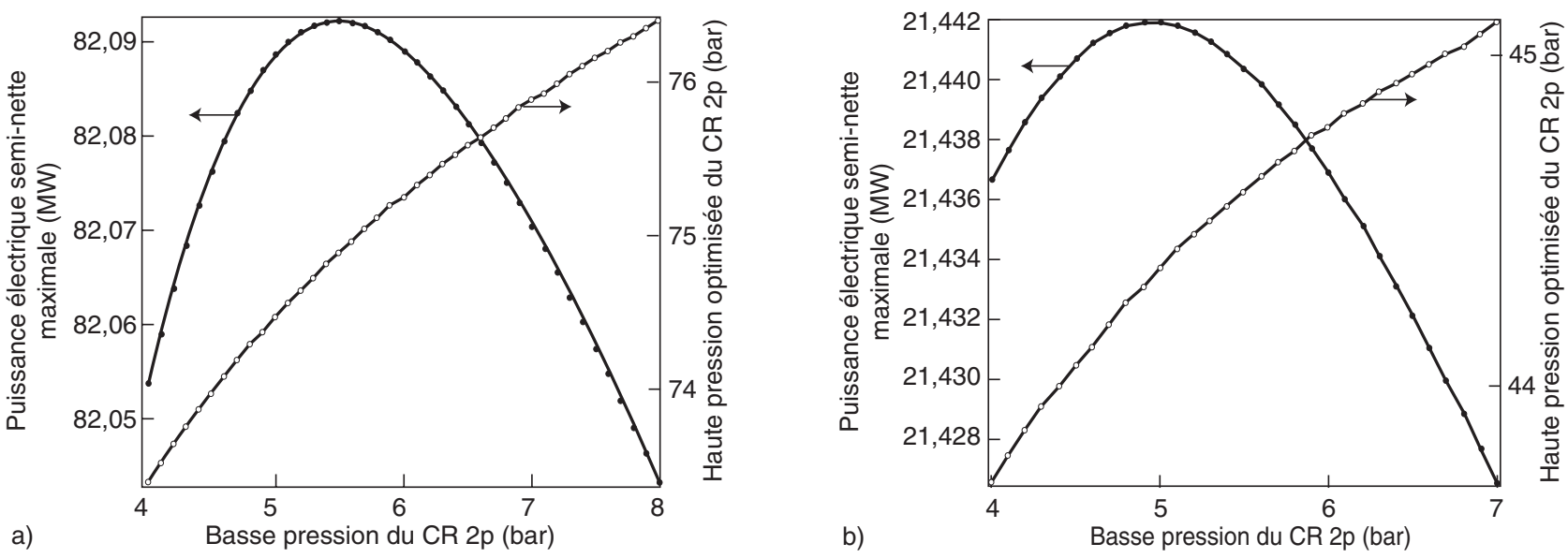

Figure 8

Optimisation CC $2 \mathrm{p}: \mathrm{P}_{\mathrm{el}}$ semi-nette maximale $=\mathrm{f}\left(\mathrm{p} 5 H_{-}\right.$optimum, $\left.\mathrm{p}_{5 \mathrm{~B}}\right)$ à gauche $; \mathrm{p}_{5 H_{-} \text {optimum }}=\mathrm{f}\left(\mathrm{p}_{5 \mathrm{~B}}\right)$ à droite $; \mathrm{p}_{5 \mathrm{~B}}$ - abscisse. a) TG : une TG de type PG9231(EC); b) TG : deux TG de type LM2500+.

The CC $2 p$ optimising: $P_{\text {el }}$ maximal output depending on the couple $\left(p_{5 H_{-} \text {optimum }}, p_{5 L}\right)$ left; $p_{5 H_{-} \text {optimum }}=f\left(p_{5 L}\right)$ right; $p_{5 L}-$ abscissa.

valeur de la $\mathrm{P}_{\mathrm{el}}$ semi-nette maximale ainsi obtenue devient un maximum des maximums des $\mathrm{P}_{\mathrm{el}}$ semi-nettes maximales. De l'analyse de la figure 8 on obtient les paires des pressions $\left(\mathrm{p}_{5 \mathrm{H} \_o p t i m u m}, \mathrm{p}_{5 \mathrm{~B} \_ \text {optimum }}\right)$ à laquelle on atteint la valeur maximum des maximums de la $\mathrm{P}_{\mathrm{el}}$ semi-nette, dans le cas CC 2p :

- pour TG stationnaires (heavy-duty) (TG: PG9231(EC)) :

$\left(\mathrm{p}_{5 \mathrm{H} \_ \text {optimum }}, \mathrm{p}_{5 \mathrm{~B} \text { optimum }}\right)=(74,9 ; 5,5)$ (bar) ;

- pour TG aerodérivative (TG: LM2500+) : ( $\mathrm{p}_{5 \mathrm{H} \_ \text {optimum, }}$ $\left.\mathrm{p}_{5 \text { B optimum }}\right)=(44,3 ; 4,9)$ (bar).

On observe que les pressions optimales dans le cas des TG stationnaires sont plus grandes que dans les cas de TG aerodérivative (figs 6 à 8). Ceci s'explique par le fait que dans le cas d'utilisation de TG aerodérivative la température à l'entrée dans la $\mathrm{CR}$ est plus faible $\left(\mathrm{t}_{6}=\mathrm{t}_{6 \mathrm{H}}=510\left({ }^{\circ} \mathrm{C}\right)\right)$ que dans le cas d'utilisation d'une TG stationnaire $\left(\mathrm{t}_{6}=\mathrm{t}_{6 \mathrm{H}}=\right.$ $558\left({ }^{\circ} \mathrm{C}\right)$ ). La conclusion qui résulte de cette analyse (et des autres cas analysés) est que si la température à l'entrée dans la CR (la sortie de la turbine à gaz) est plus petite, alors les valeurs des pressions optimales sont plus petites.

Aussi, la puissance aux bornes d'alternateur $\mathrm{P}_{\mathrm{b}}$ s'optimise, mais les valeurs des pressions optimales sont plus grandes que les valeurs des pressions pour lesquelles s'optimise la $\mathrm{P}_{\mathrm{el}}$ semi-nette, en considérant les mêmes conditions de fonctionnement. La justification de ce fait est que la variation de la puissance demandée par PA est croissante de façon monotone avec la pression de refoulement de la pompe, et donc, 
tient compte des pertes de pression dans la CR, avec la pression de la vapeur à la sortie de la $\mathrm{CR}$. $\mathrm{Si}$ on ajoute à une courbe $\left(\mathrm{P}_{\mathrm{el}}\right.$ semi-nette) qui se maximise la courbe de puissance reçue par PA monotone croissante la courbe $(\mathrm{Pb})$ résultante aura l'optimum déplacé à droite.

Les valeurs obtenues pour $\mathrm{p}_{5 \mathrm{~B} \_o p t i m u m}$ justifient les hypothèses faites sur la pression de la vapeur à l'entrée dans la section BP $\left(\mathrm{p}_{17}\right)$ de la TV et sur la possibilité de dégazage au ballon BP de la $C R\left(p_{2 B}=p_{3 B}\right)$ pour le cas de CC $2 p$. Les pressions $\mathrm{p}_{17}$ et $\mathrm{p}_{3 \mathrm{~B}}$ sont un peu plus petites que la $\mathrm{p}_{5 \mathrm{~B} \_ \text {optimum }}$ à cause des pertes de pression dans les tuyaux de liaison entre la $\mathrm{CR}$ et la TV, respectivement dans le surchauffeur basse pression $\left(\mathrm{SC}_{\mathrm{BP}}\right)$.

Pour les cas étudiés et pour les pressions optimales obtenues (figs 6 à 8), le titre de la vapeur à la sortie de la TV est dans les limites données de l'érosion des aubages, dépassant la valeur de 88 (\%) (fig. 9). On mentionne que le titre doit être supérieur à $86(\%)$.

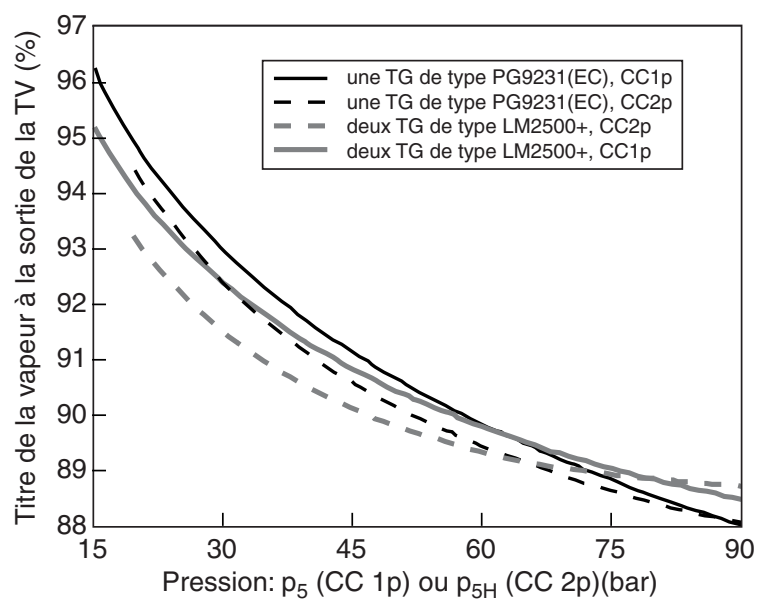

Figure 9

CC $2 p$ : Variation du titre de la vapeur à la sortie de la TV fonction de $\mathrm{p}_{5}$ (CC 1p) ou $\mathrm{p}_{5 \mathrm{H}}$ (CC $2 \mathrm{p}$ ) (abscisse), du type de TG et du type de CC.

CC $2 p$ : Output steam quality depending on $p_{5}(C C 1 p)$ or $p_{5 H}$ (CC 2p) (abscissa), on GT model and CC design.

On mentionne que les valeurs choisies pour le produit $\mathrm{S}_{\mathrm{CR}} \cdot \mathrm{k}_{\mathrm{ECO}}$ (tableau 1), ont conduit aux valeurs du pincement, dans la CR, dans l'intervalle fourni par l'expérience des fabricants de CR, pour les cas étudiés [7].

TABLEAU

Les valeurs choisies pour $\mathrm{S}_{\mathrm{CR}} \mathrm{k}_{\mathrm{ECO}}\left(\mathrm{kW} .{ }^{\circ} \mathrm{C}^{-1}\right)$

The choose data for $S_{C R} k_{E C O}\left(k W .{ }^{\circ} C^{-1}\right)$

\begin{tabular}{l|c|c}
\hline & CC 1p & CC 2p \\
\hline PG9231(EC) & 3500 & 5000 \\
\hline LM2500+ & 500 & 700 \\
\hline
\end{tabular}

Si on compare les valeurs de $\mathrm{P}_{\mathrm{el}}$ semi-nettes maximales obtenues dans le cas du CC $2 \mathrm{p}$ avec le cas du CC 1p, aux pressions optimales, on remarque que :

- Dans le cas du CC avec TG stationnaires (PG9231(EC)), la $\mathrm{P}_{\mathrm{el}}$ semi-nette augmente de 75,05 (MW), dans le cas du CC 1p, à 82,09 (MW), dans le cas de CC 2p, l'augmentation en pourcentage étant de 9,38 (\%), SCR augmentant de 42,86 (\%).

- Dans le cas du CC avec TG «aerodérivative» $(2 *$ LM2500+) la $\mathrm{P}_{\mathrm{el}}$ semi-nette augmente de 19,56 (MW), dans le cas du CC 1p, à 21,44 (MW), dans le cas de CC 2p, l'augmentation en pourcentage étant de 9,61 (\%), SCR augmentant de 40 (\%).

Ces augmentations sont significatives.

\section{MODÈLE POUR L'OPTIMISATION ÉCONOMIQUE DE LA CR IP}

Les puissances thermiques transférées par les surfaces de la CR 1p peuvent être écrites en utilisant les relations suivantes :

$$
\begin{gathered}
\dot{Q}_{E C O}=k_{E C O} S_{E C O} \Delta t_{m \ln , E C O} \\
\dot{Q}_{V A P}=k_{V A P} S_{V A P} \Delta t_{m \ln , V A P} \\
\dot{Q}_{S C}=k_{S C} S_{S C} \Delta t_{m \ln , S C}
\end{gathered}
$$

Utilisant les équations (8) à (10) et tenant compte des hypothèses de calcul considérées $\left(\mathrm{k}_{\mathrm{ECO}}=\mathrm{k}_{\mathrm{VAP}}\right.$ et $\mathrm{f}_{\mathrm{SC}}=\mathrm{k}_{\mathrm{ECO}} /$ $\left.\mathrm{k}_{\mathrm{SC}}\right)$, la relation de calcul pour le produit $\mathrm{S}_{\mathrm{CR}} \cdot \mathrm{k}_{\mathrm{ECO}}(\mathrm{CR} 1 \mathrm{p})$ est :

$$
\begin{aligned}
& S_{C R} k_{E C O}=\left(S_{E C O}+S_{V A P}+S_{S C}\right) k_{E C O} \\
= & \frac{\dot{Q}_{E C O}}{\Delta t_{m \ln , E C O}}+\frac{\dot{Q}_{V A P}}{\Delta t_{m \ln , V A P}}+f_{S C} \frac{\dot{Q}_{S C}}{\Delta t_{m \ln , S C}}
\end{aligned}
$$

On calcule le quota des surfaces d'échange de chaleur (ECO, VAP, SC) de la surface totale de la CR (12 à 14), utilisant les équations (8) à (10) et tenant compte des hypothèses de calcul $\left(\mathrm{k}_{\mathrm{ECO}}=\mathrm{k}_{\mathrm{VAP}}\right.$ et $\left.\mathrm{f}_{\mathrm{SC}}=\mathrm{k}_{\mathrm{ECO}} / \mathrm{k}_{\mathrm{SC}}\right)$ :

$$
\begin{gathered}
P S_{E C O}=\frac{S_{E C O}}{S_{C R}}=\frac{\dot{Q}_{E C O}}{\left(S_{C R} k_{E C O}\right) \Delta t_{m \ln , E C O}} \\
P S_{V A P}=\frac{S_{V A P}}{S_{C R}}=\frac{\dot{Q}_{V A P}}{\left(S_{C R} k_{E C O}\right) \Delta t_{m \ln , V A P}} \\
P S_{S C}=\frac{S_{S C}}{S_{C R}}=f_{S C} \frac{\dot{Q}_{S C}}{\left(S_{C R} k_{E C O}\right) \Delta t_{m \ln , S C}}
\end{gathered}
$$

Si on définit le coût spécifique de l'échangeur de chaleur C comme le rapport entre l'investissement I et la surface de transfert de chaleur S de celui-ci (15), alors les investissements dans les trois échangeurs de chaleur sont données par 
les relations de (16) à (18), où $\mathrm{C}_{\mathrm{ECO}}, \mathrm{C}_{\mathrm{VAP}}$ et $\mathrm{C}_{\mathrm{SC}}$ représente les coûts spécifique de l'ECO, de VAP et pour SC; l'investissement pour la $\mathrm{CR}$ résulte de la somme des investissements partiels (19):

$$
\begin{gathered}
C=\frac{I}{S} \\
I_{E C O}=S_{E C O} C_{E C O} \\
I_{V A P}=S_{V A P} C_{V A P} \\
I_{S C}=S_{S C} C_{S C} \\
I_{C R}=I_{E C O}+I_{V A P}+I_{S C}= \\
=S_{E C O} C_{E C O}+S_{V A P} C_{V A P}+S_{S C} C_{S C}
\end{gathered}
$$

En rapportant les investissements partiels (16 à 18) à l'investissement total (19) on obtient le quota de l'investissement de chacun de ces trois échangeurs de chaleur (20 à 22) :

$$
\begin{gathered}
P I_{E C O}=\frac{I_{E C O}}{I_{C R}} \\
=\frac{S_{E C O} C_{E C O}}{S_{E C O} C_{E C O}+S_{V A P} C_{V A P}+S_{S C} C_{S C}} \\
=\frac{P I_{V A P}=\frac{I_{V A P}}{I_{C R}}}{S_{E C O} C_{E C O}+S_{V A P} C_{V A P}+S_{S C} C_{S C}} \\
=\frac{S_{V A P} C_{V A P}=\frac{I_{S C}}{I_{C R}}}{S_{E C O} C_{E C O}+S_{V A P} C_{V A P}+S_{S C} C_{S C}}
\end{gathered}
$$

Dans ce cas, avec un seul niveau de pression pour la production de vapeur, les épaisseurs des tuyaux ECO, VAP et $\mathrm{SC}$ sont pratiquement constantes, parce que la pression varie dans ces tuyaux seulement à cause des pertes de pression due à l'écoulement dans ceux-ci, et la masse surfacique $\left(\mathrm{kg} \cdot \mathrm{m}^{-2}\right)$ (la masse de l'échangeur rapporté à l'unité de surface de celle-ci) de ces trois échangeurs de chaleurs est la même. Tenant compte du fait que tant ECO que VAP travaillent à températures inférieurs à $400^{\circ} \mathrm{C}$, on utilise le même type d'acier pour leurs réalisations et on peut considérer que le coût spécifique de l'ECO est pratiquement égal avec celui du $\operatorname{VAP}(23)$ :

$$
\mathrm{C}_{\mathrm{ECO}}=\mathrm{C}_{\mathrm{VAP}}
$$

Du fait que SC travaille à des températures élevées, pour la réalisation de celle-ci on utilise un autre type d'acier, plus cher que celui utilisé pour ECO et VAP. Ainsi on a un coût spécifique de SC plus grand que celui d'ECO et VAP. Dans le modèle on tient compte de ce fait par l'intermédiaire d'un coefficient supérieur à l'unité qui représente le rapport entre le coût spécifique de surchauffeur $\left(\mathrm{C}_{\mathrm{SC}}\right)$ et de l'économiseur $\left(\mathrm{C}_{\mathrm{ECO}}\right)(24)$ :

$$
R C=\frac{C_{S C}}{C_{E C O}}
$$

En rapportant les relations de (20) à (22) à $\mathrm{S}_{\mathrm{CR}} \cdot \mathrm{C}_{\mathrm{ECO}}$, $\mathrm{S}_{\mathrm{CR}} \cdot \mathrm{C}_{\mathrm{VAP}}$, respectivement à $\mathrm{S}_{\mathrm{CR}} \cdot \mathrm{C}_{\mathrm{SC}}$ et tenant compte de (23) et (24) on obtient les relations de (25) à (27). Ces dernières sont utilisées dans le modèle pour le calcul des quotas de l'investissement dans ECO (25), VAP (26) et SC (27) par rapport à l'investissement total dans la $\mathrm{CR}\left(\mathrm{I}_{\mathrm{CR}}\right)$ :

$$
\begin{gathered}
P I_{E C O}=\frac{P S_{E C O}}{P S_{E C O}+P S_{V A P}+P S_{S C} R C} \\
P I_{S V}=\frac{P S_{S V}}{P S_{E C O}+P S_{V A P}+P S_{S C} R C} \\
P I_{E C O}=\frac{P S_{S C}}{\frac{P S_{E C O}}{R C}+\frac{P S_{V A P}}{R C}+P S_{S C}}
\end{gathered}
$$

\section{OPTIMISATION ÉCONOMIQUE DU CR IP. ANALYSE DES RÉSULTATS OBTENUS}

On a tracé, en fonction de la pression $\mathrm{p}_{5}$, pour la turbine PG9231(EC), la variation :

- des quota de surfaces d'échange de chaleur de la CR 1p par rapport à la surface totale d'échange de chaleur (fig. 10) ;

- des quota d'investissement dans les surfaces d'échange de chaleur de la CR 1p par rapport à l'investissement total de la CR 1p (fig. 11).

Les paramètres qui caractérisent la $\mathrm{CR}$ ont les valeurs suivantes : $\mathrm{f}_{\mathrm{SC}}=1,3$ [19], le point d'approche $=10^{\circ} \mathrm{C}$ [7], $\mathrm{t}_{6}-\mathrm{t}_{5}=30^{\circ} \mathrm{C}[15], \mathrm{t}_{1}=\mathrm{t}_{\text {ref }}=35^{\circ} \mathrm{C}[15,23]$, RC étant considéré $=2,2$.

$\mathrm{PS}_{\mathrm{VAP}}$ décroît à l'augmentation de $\mathrm{p}_{5}$, dû à la décroissance de la chaleur latente de vaporisation avec la croissance de $\mathrm{p}_{5}$ (fig. 10).

Il existe un optimum économique, qui est mis en évidence par la minimisation du quota de surface d'échange de chaleur avec le plus grand coût spécifique $\left(\mathrm{C}_{\mathrm{SC}}\right)$, pour une surface totale d'échange de chaleur de CR imposée (fig. 10).

Néanmoins, $\mathrm{PS}_{\mathrm{SC}}$ et $\mathrm{PI}_{\mathrm{SC}}$ varient très peu entre les pressions optimisées du point de vue économique (20 bar) et thermodynamique (35 bar), pour une TG de type stationnaires. Cela est dû au fort aplatissement de ces courbes sur cette zone (figs 10 et 11). Dans le cas de TG de type 


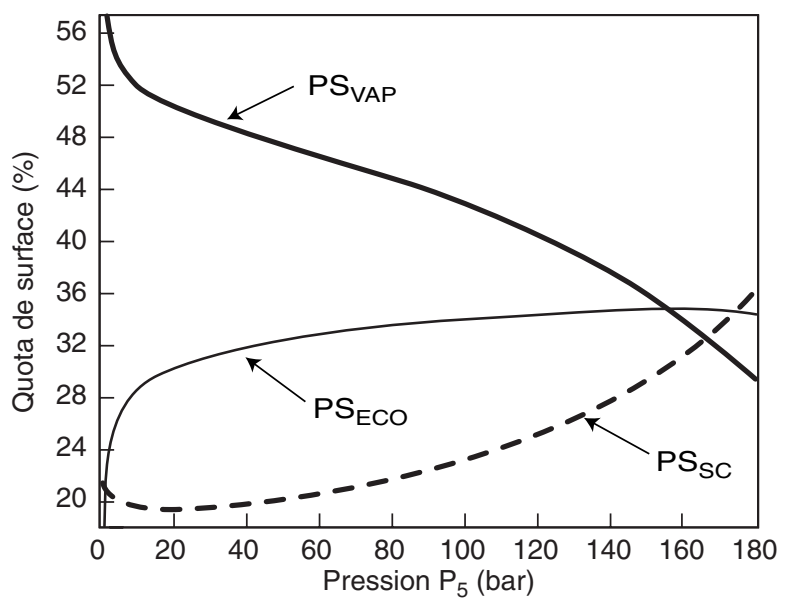

Figure 10

Le quota des surfaces d'échange de chaleur dans la CR fonction de $\mathrm{p}_{5}$, pour PG9231(EC).

The quota of heat exchange areas of HRSG depending on $p_{5}$ for $P G 9231(E C)$.

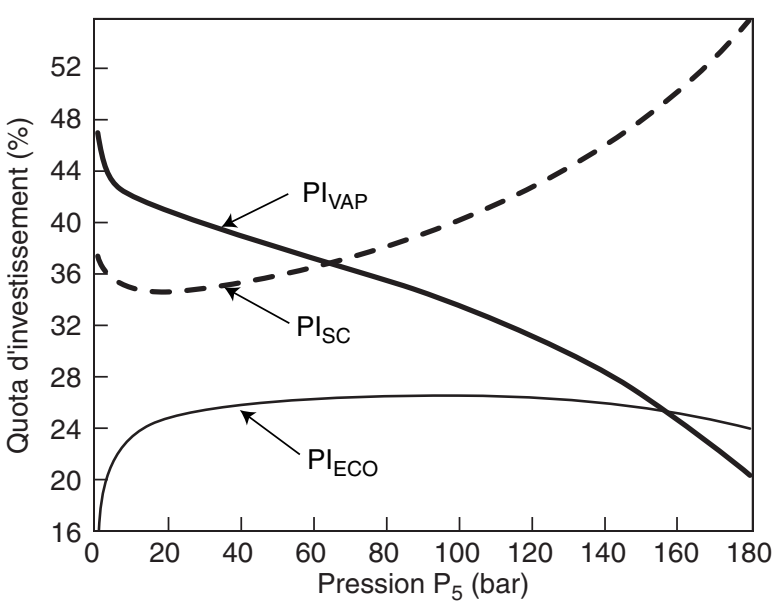

Figure 11

Le quota d'investissement dans les surfaces d'échange de chaleur de la CR fonction de $\mathrm{p}_{5}$, pour PG9231(EC).

The quota of investment in heat exchange areas of HRSG depending on $p_{5}$ for $P G 9231(E C)$. stationnaires, entre 20 bar et 35 bar, $\mathrm{PS}_{\mathrm{SC}}$ varie entre 19,32\% et $19,64 \%$ donc avec $1,66 \%$ d'écart relatif et $\mathrm{PI}_{\mathrm{SC}}$ varie avec $1,37 \%$ d'écart relatif.

En conclusion, on observe une variation très petite de $\mathrm{PS}_{\mathrm{SC}}$ et de $\mathrm{PI}_{\mathrm{SC}}$, entre les pressions qui donnent l'optimum économique et l'optimum thermodynamique (optimum de puissance électrique), dans l'hypothèse $S_{C R}$ constant.

On remarque, aussi, les faibles valeurs de $\mathrm{PS}_{\mathrm{SC}}$ en comparaison avec $\mathrm{PS}_{\mathrm{ECO}}$ et $\mathrm{PS}_{\mathrm{VAP}}$. Il en résulte une variation très faible de l'investissement dans la CR pour l'intervalle de pression et pour les hypothèses considérées.

\section{CONCLUSIONS}

Les auteurs ont fait la modélisation des cycles combinés gaz/vapeur avec des chaudières récupératrices ayant un ou deux niveaux de pression du vapeur, sans resurchauffe, en vue de l'optimisation thermodynamique (maximisation de la puissance électrique produite).

D'autre part l'optimisation thermodynamique prend en considération les aspects économiques du aux hypothèses originales de calcul (surface de transfert de chaleur imposée).

Deux types de turbine à gaz ont été employés dans les calculs, stationnaire et dérivée d'aviation, pour mettre en évidence les différentes valeurs optimales des pressions pour lesquelles on obtient des puissances électriques maximales du cycle combiné.

L'optimum économique est mis en évidence par la minimisation du quota de surface d'échange du surchauffeur (qui a le plus grand coût spécifique) par rapport à la surface totale d'échange de la chaudière $\left(\mathrm{PS}_{\mathrm{SC}}\right)$ et du quota de l'investisse- ment du surchauffeur par rapport à l'investissement total de la chaudière $\left(\mathrm{PI}_{\mathrm{SC}}\right)$. On a remarque que la courbe qui donne la variation de la $\mathrm{PS}_{\mathrm{SC}}$ et de la $\mathrm{PI}_{\mathrm{SC}}$ varie peu entre les pressions optimales résultant dans les deux types d'optimisation.

\section{BIBLIOGRAPHIE}

1 Subrahmanyam, N.V.R.S.S., Rajaram, S. et Kamalanathan, N. (1995) HRSG's for Combined Cycle Power Plants. Heat Recovery Systems \& CHP, 15, 155-161.

2 Burer, M., Tanaka, K., Favrat, D. et Yamada, K. (2003) Multi-Criteria Optimization of a District Cogeneration Plant Integrating a Solid Oxide Fuel Cell-Gas Turbine Combined Cycle, Heat Pumps and Chillers. Energy, 28, 497-518.

3 Carcasci, C. et Facchini, B. (2000) Comparison between Two Gas Turbine Solutions to Increase Combined Power Plant Efficiency. Energy Conversion and Management, 41, 757773.

4 Kim, T.S. et Ro., S.T. (2000) Power Augmentation of Combined Cycle Power Plants Using Cold Energy of Liquefied Natural Gas. Energy, 25, 841-856.

5 Liszka, M., Manfrida, G. et Ziebik, A. (2003) Parametric Study of HRSG in Case of Repowered Industrial CHP Plant. Energy Conversion and Management, 44, 995-1012.

6 Najjar, Y.S.H. (1999) Comparison of Performance of the Integrated Gas and Steam Cycle (IGSC) with the Combined Cycle (CC). Applied Thermal Engineering, 19, 75-87.

7 Shin, J.Y., Jeon, Y.J., Maeng, D.J., Kim, J.S. et Ro, S.T. (2002) Analysis of the Dynamic Characteristics of a Combined-Cycle Power Plant. Energy, 27, 1085-1098.

8 Traverso, A. et Massardo, A.F. (2002) Thermoeconomic Analysis of Mixed Gas-Steam Cycles. Applied Thermal Engineering, 22, 1-21.

9 Valdés, M. et Rapún, J.L. (2001) Optimisation of Heat Recovery Steam Generators for Combined Cycle Gas Turbine Power Plants. Applied Thermal Engineering, 21, 1149-1159. 
10 Franco A. et Russo, A. (2002) Combined Cycle Plant Efficiency Increase Based on the Optimization of the Heat Recovery Steam Generator Operating Parameters. International Journal of Thermal Sciences, 41, 843-859.

11 Casarosa, C., Donatini, F. et Franco, A. (2002) Thermoeconomic Optimization of Heat Recovery Steam Generators Operating Parameters for Combined Plants. Energy, In press, Corrected Proof, Available online 2 December 2002.

12 Valdés, M., Durán, D. et Rovira, A. (2003) Thermoeconomic Optimization of Combined Cycle Gas Turbine Power Plants Using Genetic Algorithms. Applied Thermal Engineering, 23, 2169-2182.

13 Alexe, F.l. et Cenusa, V. (2002) Optimizarea parametrilor la sursa calda ciclului cu abur din CNE (PWR si PHWR) prin metode exergetice. Producerea, transportul si distributia energiei electrice si termice, 5-6.

14 Monteil, J.M. (2003) Centrale à cycle combiné. Composants potentiels. Techniques de l'ingénieur, traité Génie énergétique, BE 3, BE8906, 1-18.

15 Darie, G., Dupleac, M., Sava M. et Cenusa, V. (2001) Cicluri combinate gaze-abur, Agir, Bucuresti.

16 Cenusa, V. (2004) Contribution à l'amélioration du couplage thermodynamique entre l'installation de la turbine à gaz et l'installation de la turbine à vapeur dans les centrales électriques à cycles combinés gaz/vapeur, Thèse de Doctorat, Université Henri Poincaré, Nancy-I, Faculté des Sciences \& Techniques, France, 25.10.2004.

17 Cenusa, V., Feidt, M., Benelmir, R. et Badea, A. (2004) Exergetic Optimization of the Heat Recovery Steam Generator by Imposing the Total Heat Transfer Area. International Journal of Thermodynamics, 7, 149-156.

18 Bontemps, A., Garrigue, A., Goubier, C., Marvillet, J., Mercier P. et Vidil, R. (1998) Technologie des échangeurs thermiques. Techniques de l'ingénieur, Paris.
19 Dumont, M.N. et Heyen, G. (2001) Mathematical Modelling of an Advanced Once-Through Sub-or Supercritical Heat Recovery Steam Generator. Power Generation and Sustainable Development, 8-9 October, Liège, Belgium.

20 Sacadura, J.F. (coordonateur) (1980) Initiation aux transferts thermiques, Technique et Documentation, Paris.

21 Dupleac, M. (1997) Perspective si optimizari pentru introducerea ciclului mixt cu amestec de gaze si abur, Thèse de Doctorat, Université Politehnica de Bucarest, România.

22 Paren, J. et Parietti, C. (1991) Centrales à cycles combines VEGA 109F ; 3 pressions de resurchauffe. Revue Technique GEC ALSTHOM, 4, 15-26.

23 Cenusa, V., Benelmir, R., Feidt, M. et Badea, A. (2003) L'optimisation exergétique des pressions de production des vapeurs dans chaudières récupératrices avec un ou deux niveaux de pression pour CC sans resurchauffe et condition de surface de transfert de la chaleur imposée. CIEM, 22-25 Octombrie, Bucuresti, România, 6.1-6.6.

24 Kostiuk, Frolov (1985) Parovaie i gazovaie turbini, GosEnergoIzdat, Moscova .

25 Alexe, Fl., Cenusa, V., Beldimanescu, Gh. et Bidina, D. (2001) Metodologii, algoritmi, functii si proceduri pentru modelarea variatiei proprietatilor termodinamice ale apei si aburului la presiuni subcritice. Producerea, transportul si distributia energiei electrice si termice, novembre-décembre, 36-40.

26 Cenusa, V., Benelmir, R., Feidt, M. et Badea, A. (2003) On Gas Turbines and Combined Cycles. International Journal of Energy, Environment and Economics, 11, 15-26.

27 Cenusa, V., Benelmir, R., Feidt, M. et Badea, A. (2004) A Model for a Gas Turbine Installation. International Journal of Energy, Environment and Economics, 12, 65-86.

Final manuscript received in January 2006

Copyright $(\subseteq) 2006$ Institut français du pétrole

Permission to make digital or hard copies of part or all of this work for personal or classroom use is granted without fee provided that copies are not made or distributed for profit or commercial advantage and that copies bear this notice and the full citation on the first page. Copyrights for components of this work owned by others than IFP must be honored. Abstracting with credit is permitted. To copy otherwise, to republish, to post on servers, or to redistribute to lists, requires prior specific permission and/or a fee: Request permission from Documentation, Institut français du pétrole, fax. +33147527078 , or revueogst@ifp.fr. 